\title{
Quantum Super-Integrable Systems as Exactly Solvable Models ${ }^{\star}$
}

\author{
Allan P. FORDY \\ Department of Applied Mathematics, University of Leeds, Leeds LS2 9JT, UK \\ E-mail: allan@maths.leeds.ac.uk \\ URL: http://www.maths.leeds.ac.uk/cnls/research/fordy/fordy.html
}

Received November 14, 2006, in final form February 05, 2007; Published online February 14, 2007

Original article is available at http://www.emis.de/journals/SIGMA/2007/025/

\begin{abstract}
We consider some examples of quantum super-integrable systems and the associated nonlinear extensions of Lie algebras. The intimate relationship between superintegrability and exact solvability is illustrated. Eigenfunctions are constructed through the action of the commuting operators. Finite dimensional representations of the quadratic algebras are thus constructed in a way analogous to that of the highest weight representations of Lie algebras.
\end{abstract}

Key words: quantum integrability; super-integrability; exact solvability; Laplace-Beltrami

2000 Mathematics Subject Classification: 35Q40; 70H06

Dedicated to the memory of Vadim Kuznetsov

\section{Introduction}

In classical mechanics, there is a well defined meaning to the term 'complete integrability'. If, in $n$ degrees of freedom, we have $n$ independent functions in involution (mutually Poisson commuting), then the system can be integrated, 'up to quadrature'. This is known as Liouville's Theorem. It is customary to consider one of these functions as 'the Hamiltonian' $H$, with the others being its first integrals and this Hamiltonian is said to be completely integrable in the Liouville sense. Whilst $n$ is the maximal number of independent functions which can be in involution, it is possible to have further integrals of the Hamiltonian $H$, which necessarily generate a non-Abelian algebra of integrals of $H$. The maximal number of additional independent integrals is $n-1$, since the 'level surface' of $2 n-1$ integrals (meaning the intersection of individual level surfaces) is just the (unparameterised) integral curve. Well known elementary examples are the isotropic harmonic oscillator, the Kepler system and the Calogero-Moser system. The quadratures of complete integrability are often achieved through the separation of variables of the Hamilton-Jacobi equation. The solution of a maximally super-integrable system can also be calculated purely algebraically (albeit implicitly), requiring just the solution of the equations $I_{k}=c_{k}, k=1, \ldots, 2 n-1$.

In $n$-dimensions, Quantum integrable systems are defined analogously by requiring the existence of $n$ mutually commuting differential operators (usually with some requirements, such as self-adjointness). One of these will be of Schrödinger type, being the quantum version of the Hamiltonian $H$. However, we don't have a theorem analogous to Liouville's and we don't have anything resembling 'reduction to quadrature'. There is a notion of separation of variables (this time in the linear PDE sense), requiring a solution to be expressible as the product of $n$ functions of a single variable. This reduces the problem of solving one Schrödinger equation in

\footnotetext{
${ }^{\star}$ This paper is a contribution to the Vadim Kuznetsov Memorial Issue "Integrable Systems and Related Topics". The full collection is available at http://www.emis.de/journals/SIGMA/kuznetsov.html
} 
$n$-dimensions to that of solving $n$ Schrödinger equations in 1-dimension. This achieves less than quadratures.

What we would like to achieve in quantum mechanics is to build the spectrum and corresponding eigenfunctions for Schrödinger's equation with some given boundary conditions. This class of Schrödinger equation is called exactly solvable. Even in 1-dimension exactly solvable Schrödinger equations are rare, so unlikely to be the end-product of applying the separation of variables method. One particular class of 1-dimension exactly solvable Schrödinger equations was presented by Infeld and Hull [8], related to the factorisation of operators. This can be related to Darboux transformations, which can then be generalised to higher dimensions (where factorisation is not possible). Darboux transformations can then be used to build exactly solvable Schrödinger equations in higher dimensions. In [4], Darboux transformations have been shown to be related to the symmetries of the Laplacian.

In the quantum case we also have a notion of super-integrability. By analogy with the classical case, super-integrability again involves having 'extra' commuting operators. Once again, we cannot have more than $n$ such operators in involution, so the algebra is necessarily non-Abelian. Furthermore, if the operators are second order, their commutators are third order, with further commutators being fourth order and so on, so cannot be expected to generate a finite dimensional Lie algebra. However, they can generate a finite dimensional algebra with nonlinear commutation relations (see [2, 10, 13], for example). There are many papers on super-integrable systems (both classical and quantum). Particularly relevant to the present paper is a series of papers by Kalnins et al. (see $[9,10]$ and references therein), in which a classification is given of super-integrable systems in 2-dimensional flat and constant curvature spaces and in which a pair of quadratic first integrals, together with their quadratic commutation relations are derived. As is well known, super-integrable systems are separable in more than one coordinate system. In most of these papers the main emphasis is on the separation of variables, first by constructing and classifying coordinate systems and corresponding 'separable potentials' and then using this (in the quantum case) to build eigenfunctions.

In the present paper, there is no discussion of separation of variables and 'differential equations techniques' are kept to a minimum. Our main emphasis is on the direct relationship between super-integrability and exactly solvability. We use the commuting operators in a direct and explicit manner to build the eigenfunctions for our operators. This construction is the analogue (in the context of our quadratic algebras) to that of the highest weight representation of simple Lie algebras (see the Appendix). In particular, the polynomial eigenfunctions of the Krall-Sheffer operator of Example 9 can be explicitly constructed in this way. The issue of the relationship between super-integrability and exact solvability is not new and is discussed in $[10,11,14]$. In [14] it is conjectured that exactly solvability should hold quite widely for super-integrable systems. Whilst operator algebras are discussed in these papers they are not used to construct eigenfunctions.

In this paper we consider some particular super-integrable systems, associated with a particular Laplace-Beltrami operator in 2-dimensions. One of the examples is in the Krall-Sheffer classification of 'admissible operators'. The relationship between Krall-Sheffer operators and super-integrability was shown by Harnad, et al in $[6,15]$. In these papers they reworked the Krall-Sheffer classification and showed that in each case the leading order terms in the operator correspond to the Laplace-Beltrami operator of either a zero or constant curvature, 2-dimensional space. They also show that each of the 9 cases is super-integrable by presenting pairs of commuting, second order operators. Example 9 of the current paper is exactly Case II of [6, 15]. In [15] it is pointed out that this case had been previously introduced and studied in [1, 10].

In the next section, we give some basic formulae related to our particular metric and its symmetries. We then present 3 super-integrable examples, with their operator algebras and finite representation spaces (families of eigenfunctions). We then give some concluding remarks. 


\section{$2 \quad$ A metric and its symmetries}

For an $n$-dimensional (pseudo-)Riemannian space, with local coordinates $x^{1}, \ldots, x^{n}$ and metric $g_{i j}$, the Laplace-Beltrami operator is defined by $L_{b} f=g^{i j} \nabla_{i} \nabla_{j} f$, which has explicit form

$$
L_{b} f=\sum_{i, j=1}^{n} \frac{1}{\sqrt{g}} \frac{\partial}{\partial x^{j}}\left(\sqrt{g} g^{i j} \frac{\partial f}{\partial x^{i}}\right),
$$

where $g$ is the determinant of the matrix $g_{i j}$. The coefficients of leading order terms in the Laplace-Beltrami operator are the coefficients of the inverse metric $g^{i j}$. For a metric with isometries, the infinitesimal generators (Killing vectors) are just first order differential operators which commute with the Laplace-Beltrami operator (1). When the space is either flat or constant curvature, it possesses the maximal group of isometries, which is of dimension $n(n+1) / 2$. In this case, $L_{b}$ is actually the second order Casimir function of the symmetry algebra (see [5]).

In this paper, we consider one particular constant curvature metric in 2-dimensions, with inverse

$$
g^{i j}=\left(\begin{array}{cc}
x^{2} & x y \\
x y & y^{2}-y
\end{array}\right) .
$$

The choice of coordinates here is motivated by the relationship to Krall-Sheffer operators [12], but otherwise quite arbitrary.

A convenient basis of Killing vectors is

$$
\mathbf{H}=4 x \partial_{x}, \quad \mathbf{E}=2 \sqrt{x y} \partial_{y}, \quad \mathbf{F}=4 \sqrt{x y} \partial_{x}+2(y-1) \sqrt{\frac{y}{x}} \partial_{y},
$$

satisfying the standard commutation relations of $s l(2, \mathbb{C})$ :

$$
[\mathbf{H}, \mathbf{E}]=2 \mathbf{E}, \quad[\mathbf{H}, \mathbf{F}]=-2 \mathbf{F}, \quad[\mathbf{E}, \mathbf{F}]=\mathbf{H} .
$$

The Laplace-Beltrami operator for the metric (2) is proportional to the quadratic Casimir operator:

$$
L_{b}=\frac{1}{16}\left(\mathbf{H}^{2}+2 \mathbf{E F}+2 \mathbf{F E}\right)=x^{2} \partial_{x}^{2}+2 x y \partial_{x} \partial_{y}+\left(y^{2}-y\right) \partial_{y}^{2}+\frac{3}{2} x \partial_{x}+\frac{1}{2}(3 y-1) \partial_{y} .
$$

Remark 1. The operator $L_{b}$ is invariant under an involution,

$$
\bar{x}=\frac{(y-1)^{2}}{x}, \quad \bar{y}=y .
$$

which, in fact, gives a concrete realisation of the Lie algebra automorphism

$$
\mathbf{E} \leftrightarrow \mathbf{F}, \quad \mathbf{H} \rightarrow-\mathbf{H} .
$$

This will be useful in some of the calculations below.

Second order operators, commuting with $L_{b}$, are just symmetric quadratic forms of Killing vectors. Suppose $\mathbf{K}$ is such an operator. Then we may seek functions $u$ and $v$, such that

$$
\left[L_{b}+u, \mathbf{K}+v\right]=0,
$$

which constitutes a coupled system of partial differential equations for $u$ and $v$. The solution depends upon a pair of arbitrary functions, each of one variable, typical of separable systems. Requiring that there exist two of these second order commuting operators, $I_{j}=\mathbf{K}_{j}+v_{j}$, strongly 
constrains these (formerly) arbitrary functions, which reduce to rational functions depending upon only a finite number of parameters. The coefficients of the second order derivatives in such an operator $\mathbf{K}$ define a contravariant, rank-two Killing tensor. For brevity, we will refer to these operators as Killing tensors in what follows.

The examples discussed below were presented in [4], in the context of Darboux transformations, which will not be discussed in this paper. Instead we discuss how to use the commuting second order operators to build eigenfunctions of $L=L_{b}+u$ in a way analogous to the highest weight representation of a Lie algebra.

For some calculations, such as those of [4], it is convenient to use separation coordinates. In our case we can choose 2 (or even 3 ) such coordinate systems, but the current calculations are as easily done in terms of the $x-y$ coordinates.

\section{The system with Killing tensors $\mathrm{H}^{2}$ and $\mathrm{E}^{2}$}

Requiring both $I_{1}=\mathbf{H}^{2}+v_{1}$ and $I_{2}=\mathbf{E}^{2}+v_{2}$ to commute with $L$ leads to the specific forms of $u(x, y), v_{i}(x, y)$ in the operators below

$$
\begin{aligned}
& L=L_{b}+\frac{c_{0}}{x}+\frac{c_{1}}{y}+c_{2} \frac{y-1}{x^{2}}, \\
& I_{1}=\mathbf{E}^{2}-4\left(c_{1} \frac{x}{y}+c_{2} \frac{y}{x}\right), \\
& I_{2}=\mathbf{H}^{2}-16\left(c_{0}\left(\frac{y-1}{x}\right)+c_{2}\left(\frac{y-1}{x}\right)^{2}\right) .
\end{aligned}
$$

In order to build eigenfunctions of $L$ it is convenient to change gauge:

$$
L \mapsto \tilde{L}=G^{-1} L G \quad \text { with } \quad G=\exp \left(\frac{\alpha}{2}\left(\frac{y-1}{x}\right)\right) x^{(\beta+\gamma-1) / 2} y^{-(2 \gamma+1) / 4},
$$

which simultaneously reduces the 3 potential terms to constants. The specific coefficients in $G$ were chosen to simplify the final expressions for the operators. Removing the additive constants and adjusting overall multiplicative factors (and dropping the 'tilde') we arrive at the following operators:

$$
\begin{aligned}
& L=x^{2} \partial_{x}^{2}+2 x y \partial_{x} \partial_{y}+\left(y^{2}-y\right) \partial_{y}^{2}+(\beta x+\alpha) \partial_{x}+(\beta y+\gamma) \partial_{y}, \\
& I_{1}=x y \partial_{y}^{2}+(\alpha y-\gamma x) \partial_{y}, \\
& I_{2}=x^{2} \partial_{x}^{2}+((\beta+\gamma) x+\alpha(1-y)) \partial_{x},
\end{aligned}
$$

where $c_{0}=\alpha(2-\beta-\gamma) / 2, c_{1}=(2 \gamma+1)(2 \gamma+3) / 16, c_{2}=\alpha^{2} / 4$. This is an example of admissible operator, as defined by Krall and Sheffer [12]. The connection between Krall-Sheffer operators and super-integrability was first pointed out in [6, 15]. Indeed, Example 9 above is exactly Case II of $[6,15]$. As pointed out in [15], this case was previously introduced and studied in $[1,10]$.

The operator $I_{3}=\left[I_{1}, I_{2}\right]$ is third order, so cannot be written as a polynomial (in particular, linear, Lie algebraic) expression in $L, I_{1}, I_{2}$, but does satisfy the polynomial equation:

$$
\begin{aligned}
I_{3}^{2}= & \frac{4}{3}\left(I_{1}^{2} I_{2}+I_{1} I_{2} I_{1}+I_{2} I_{1}^{2}-2 I_{1}^{2}\right)+\alpha(\beta-\gamma-2)\left(I_{1} I_{2}+I_{2} I_{1}-\frac{4}{3} I_{1}\right) \\
& +(\beta+\gamma-2)\left((\beta+\gamma) I_{1}^{2}-2 \alpha I_{1} L\right)+\alpha^{2}\left(I_{2}-L\right)^{2}+\frac{2}{3} \alpha^{2}\left(I_{2}-(3 \gamma+1) L\right) .
\end{aligned}
$$


These 4 operators satisfy the commutation relations:

$$
\begin{aligned}
& {\left[L, I_{1}\right]=\left[L, I_{2}\right]=\left[L, I_{3}\right]=0, \quad\left[I_{1}, I_{2}\right]=I_{3},} \\
& {\left[I_{1}, I_{3}\right]=2 I_{1}^{2}+\alpha(\beta-\gamma-2) I_{1}+\alpha^{2}\left(I_{2}-L\right),} \\
& {\left[I_{2}, I_{3}\right]=-2\left(I_{1} I_{2}+I_{2} I_{1}\right)+(\beta+\gamma-2)\left(\alpha L-(\beta+\gamma) I_{1}\right)-\alpha(\beta-\gamma-2) I_{2} .}
\end{aligned}
$$

By definition (see [12]), an admissible operator possesses a sequence of eigenvalues $\lambda_{n}$ (with $\lambda_{n} \neq \lambda_{m}$ for $\left.m \neq n\right)^{1}$, such that, for each $n \geq 0$, there exist $n+1$ linearly independent polynomial eigenfunctions of degree $n$, with eigenvalue $\lambda_{n}$. The degeneracy (for $n>0$ ) of $\lambda_{n}$ stems from the super-integrability in a very explicit way. We show below how to use the operators $I_{1}$ and $I_{2}$ to build sequences of polynomial eigenfunctions of $L$, using a construction which is analogous to that of the 'highest weight representation' of a simple Lie algebra.

We start with a polynomial eigenfunction

$$
P_{n, 0}=x^{n}+\sum_{i=1}^{n} \rho_{i} x^{n-i}
$$

for constants $\rho_{i}$, which is independent of $y$ and therefore in the Kernel of $I_{1}$. This satisfies the 1-dimensional eigenvalue problem

$$
L_{x} P_{n, 0} \equiv\left(x^{2} \partial_{x}^{2}+(\beta x+\alpha) \partial_{x}\right) P_{n, 0}=\lambda_{n} P_{n, 0}, \quad \lambda_{n}=n(n+\beta-1) .
$$

This value of $\lambda_{n}$ is easily determined by looking at the coefficient of $x^{n}$. It is easy to show that

$$
P_{1,0}=x+\frac{\alpha}{\beta}, \quad P_{2,0}=x^{2}+\frac{2 \alpha x}{\beta+2}+\frac{\alpha^{2}}{(\beta+2)(\beta+1)},
$$

and that this sequence satisfies the 3 -point recursion relation

$$
\mathbf{r}_{x}: \quad P_{n+1,0}=\left(x+A_{n}\right) P_{n, 0}+B_{n} P_{n-1,0},
$$

with

$$
A_{n}=\frac{\alpha(\beta-2)}{(\beta+2 n)(\beta+2 n-2)}, \quad B_{n}=\frac{\alpha^{2} n(n+\beta-2)}{(\beta+2 n-1)(\beta+2 n-2)^{2}(\beta+2 n-3)} .
$$

We now use $I_{2}$ (for $\alpha \neq 0$ ) to build a sequence of (monic) eigenfunctions

$$
P_{n-k, k}=x^{n-k} y^{k}+\text { lower order terms, } \quad k=1, \ldots, n .
$$

The case of $\alpha=0$ is the reduction $c_{0}=c_{2}=0$, which has a different algebra and is dealt with later (see the system (12)). Since $I_{2}$ commutes with $L$, all such eigenfunctions will have the same eigenvalue $\lambda_{n}$, which means (important for $P_{0, n}$, below) that the degree of each polynomial must be $n$. The polynomials $P_{n-k, k}$ are defined recursively by

$$
I_{2} P_{n-k, k}=(n-k)(n-k-1+\beta+\gamma) P_{n-k, k}-\alpha(n-k) P_{n-k-1, k+1}, \quad k=0, \ldots, n-1,
$$

as can be seen by inspecting the form of $I_{2}$ and by inspecting the coefficients of $x^{n-k} y^{k}$ and $x^{n-k-1} y^{k+1}$. It can be seen that $I_{2} P_{0, n}=0$, so the polynomial $P_{0, n}$ must be independent of $x .^{2}$ The polynomials $P_{0, n}(y)$ also satisfy a 3 -point recursion relation [3]

$$
\mathbf{r}_{y}: \quad P_{0, n+1}=\left(y+\tilde{A}_{n}\right) P_{0, n}+\tilde{B}_{n} P_{0, n-1},
$$

\footnotetext{
${ }^{1}$ The condition (3.8) of [12], which ensures this, is that $\beta$ should not be a negative integer.

${ }^{2}$ Since the leading order term is killed, any non-zero result would be a polynomial of lower degree, which is not possible. This can also be seen by setting $k=n$ in (11).
} 
with

$$
\begin{aligned}
& \tilde{A}_{n}=\frac{(n+1)(\gamma-n)}{\beta+2 n}-\frac{n(\gamma-n+1)}{\beta+2 n-2} \\
& \tilde{B}_{n}=-\frac{n(\gamma-n+1)}{2(\beta+2 n-2)}\left((n+1) \frac{\gamma-n}{\beta+2 n-1}-2 n \frac{\gamma-n+1}{\beta+2 n-2}+(n-1) \frac{\gamma-n+2}{\beta+2 n-3}\right) .
\end{aligned}
$$

The space of polynomials spanned by this basis is also invariant under the action of $I_{1}$ :

$$
I_{1} P_{n-k, k}=k \alpha P_{n-k, k}+k(k-\gamma-1) P_{n-k+1, k-1}, \quad k=1, \ldots, n .
$$

This array of polynomials, together with the action of $I_{1}, I_{2}$ and the 3 -point recursion relations, are depicted in Fig. 1.

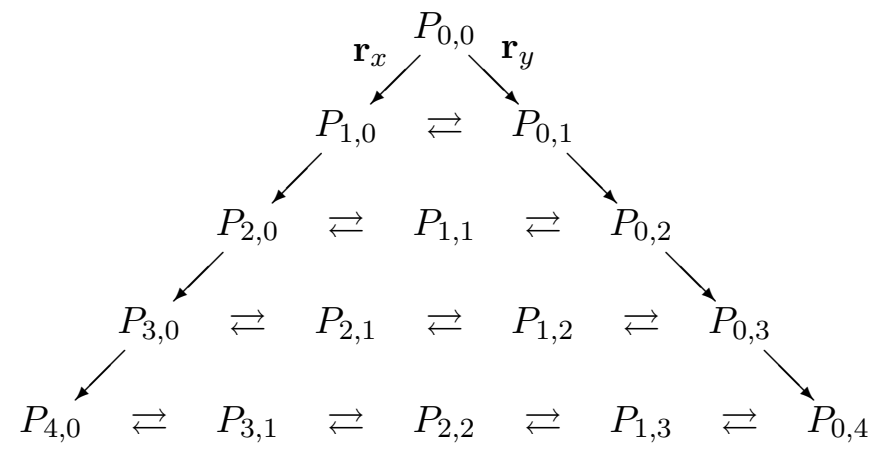

Figure 1. The triangular lattice of polynomials $P_{n-k, k}, k=0, \ldots, n$ with $P_{0,0}=1$, for system (9). Horizontal arrows denote the action of $I_{1}$ (left) and $I_{2}$ (right).

For each $n \geq 1$, the sequence of polynomials $P_{n-k, k}, k=0, \ldots, n$, defines an $n+1$ dimensional representation of the algebra (10). These can be explicitly calculated. For instance, apart from $P_{1,0}$ and $P_{2,0}$, given above, we have

$$
\begin{aligned}
& P_{0,1}=y+\frac{\gamma}{\beta}, \quad P_{1,1}=x y+\frac{1}{\beta+2}(\gamma x+\alpha y)+\frac{\alpha \gamma}{(\beta+2)(\beta+1)}, \\
& P_{0,2}=y^{2}+2 \frac{(\gamma-1) y}{\beta+2}+\frac{\gamma(\gamma-1)}{(\beta+2)(\beta+1)} .
\end{aligned}
$$

It is easy to see that, for $n \geq 2$, the 4 representative matrices generate a larger Lie algebra (commutators lie outside the linear span), but do satisfy the quadratic relations (10).

\section{The system with Killing tensors $\mathbf{H}^{2}$ and $\mathrm{F}^{2}$}

Requiring both $I_{1}=\mathbf{H}^{2}+v_{1}$ and $I_{2}=\mathbf{F}^{2}+v_{2}$ to commute with $L$ leads to the specific forms of $u(x, y), v_{i}(x, y)$ in the operators below

$$
\begin{aligned}
& L=L_{b}+c_{0} \frac{x}{(y-1)^{2}}+\frac{c_{1}}{y}+c_{2} \frac{x^{2}}{(y-1)^{3}}, \\
& I_{1}=\mathbf{F}^{2}-4\left(c_{1} \frac{(y-1)^{2}}{x y}+c_{2} \frac{x y}{(y-1)^{2}}\right), \\
& I_{2}=\mathbf{H}^{2}-16\left(c_{0}\left(\frac{x}{y-1}\right)+c_{2}\left(\frac{x}{y-1}\right)^{2}\right) .
\end{aligned}
$$


These are, in fact, directly transformed from (7) under the action of the involution (6). Either by composing the gauge transformation (8) with this involution or by directly transforming (9) by (6) (and dropping 'bars'), we obtain

$$
\begin{aligned}
L= & x^{2} \partial_{x}^{2}+2 x y \partial_{x} \partial_{y}+\left(y^{2}-y\right) \partial_{y}^{2}+\left(\beta x+\frac{2(\beta+\gamma-1) x}{y-1}-\frac{\alpha x^{2}}{(y-1)^{2}}\right) \partial_{x}+(\beta y+\gamma) \partial_{y}, \\
I_{1}= & 4 x y \partial_{x}^{2}+4 y(y-1) \partial_{x} \partial_{y}+\frac{y(y-1)^{2}}{x} \partial_{y}^{2}+2\left((1-\gamma) y+\frac{\alpha x y}{y-1}+\gamma\right) \partial_{x} \\
& +\left(\alpha y-\frac{\gamma(y-1)^{2}}{x}\right) \partial_{y} \\
I_{2}= & x^{2} \partial_{x}^{2}+\left((2-\beta-\gamma) x-\frac{\alpha x^{2}}{1-y}\right) \partial_{x}
\end{aligned}
$$

where $c_{0}=\alpha(2-\beta-\gamma) / 2, c_{1}=(2 \gamma+1)(2 \gamma+3) / 16, c_{2}=\alpha^{2} / 4$. These satisfy the same quadratic relations (10). Starting from these operators, it would not be so easy to find a simple function in the kernel of $I_{1}$, and then to build the sequence of eigenfunctions, but there is no need to do this, since the functions

$$
Q_{n-k, k}(x, y)=P_{n-k, k}\left(\frac{(y-1)^{2}}{x}, y\right)
$$

are obtained directly through the involution. The operator $L$ is no longer 'admissible' in the Krall-Sheffer sense, and the eigenfunctions are no longer polynomial, but the same set of relations hold, leading to the same diagram of Fig. 1.

\section{The system with Killing tensors $\mathrm{E}^{2}$ and $\mathrm{F}^{2}$}

Requiring both $I_{1}=\mathbf{E}^{2}+v_{1}$ and $I_{2}=\mathbf{F}^{2}+v_{2}$ to commute with $L$ leads to the specific forms of $u(x, y), v_{i}(x, y)$ in the operators below

$$
L=L_{b}+\frac{c_{1}}{y}, \quad I_{1}=\mathbf{E}^{2}-4 c_{1} \frac{x}{y}, \quad I_{2}=\mathbf{F}^{2}-4 c_{1} \frac{(y-1)^{2}}{x y} .
$$

However, it can be seen that the potential of $L$ is a subcase of (7), with $c_{0}=c_{2}=0$, so there should also be an integral of the form $I_{3}=\mathbf{H}^{2}+v_{3}$. Indeed, it should be exactly as in the case of (7). However, in this reduction, we just have $I_{3}=\mathbf{H}^{2}$, so may consider the algebra generated by $L, I_{1}, I_{2}$ and $\mathbf{H}$, which satisfy the commutation relations

$$
\left[\mathbf{H}, I_{1}\right]=4 I_{1}, \quad\left[\mathbf{H}, I_{2}\right]=-4 I_{2}, \quad\left[I_{1}, I_{2}\right]=2\left(8 c_{1}-1\right) \mathbf{H}-\mathbf{H}^{3}+16 \mathbf{H} L,
$$

as well as $\left[L, I_{1}\right]=\left[L, I_{2}\right]=[L, \mathbf{H}]=0$. Under the action of the involution (6), $L \mapsto L, I_{1} \mapsto I_{2}$, $I_{2} \mapsto I_{1}, \mathbf{H} \mapsto-\mathbf{H}$, which preserves the commutation relations (13). The Casimir operator of the algebra (13) is

$$
\mathcal{C}=I_{1} I_{2}+I_{2} I_{1}+\frac{1}{2}\left(8 c_{1}-5\right) \mathbf{H}^{2}-\frac{1}{8} \mathbf{H}^{4}+4 \mathbf{H}^{2} L .
$$

This is not, of course, independent of $L$ and can be written $\mathcal{C}=32 L^{2}-16\left(4 c_{1}+1\right) L+16 c_{1}\left(2 c_{1}-1\right)$.

We mimic the highest weight representation of $\operatorname{sl}(2, \mathbb{C})$ and seek function $\varphi_{1}(x, y)$, satisfying

$$
\mathbf{H} \varphi_{1}=s \varphi_{1}, \quad L \varphi_{1}=\lambda \varphi_{1}, \quad I_{1} \varphi_{1}=0
$$


The first of these gives $\varphi_{1}(x, y)=x^{s / 4} \psi(y)$, so the remaining equations lead to

$$
\begin{aligned}
& 16 y^{2}(y-1) \psi^{\prime}+8 y((s+3) y-1) \psi^{\prime}+\left(16 c_{1}+(s(s+2)-16 \lambda) y\right) \psi=0, \\
& 2 y^{2} \psi^{\prime}+y \psi^{\prime}-2 c_{1} \psi=0
\end{aligned}
$$

which can be solved for both $\lambda$ and $\psi$ to give

$$
\lambda_{m, s}=\frac{1}{16}(s+m+1)(s+m+3), \quad \psi=y^{(m+1) / 4}, \quad \text { where } \quad c_{1}=\frac{m^{2}-1}{16} .
$$

We now define

$$
\varphi_{k}=I_{2}^{k-1} \varphi_{1}, \quad \text { satisfying } \quad \mathbf{H} \varphi_{k}=(s-4(k-1)) \varphi_{k} .
$$

Under the involution, we have $\varphi_{1} \mapsto \bar{\varphi}_{1}$ and

$$
\mathbf{H} \bar{\varphi}_{1}=-s \bar{\varphi}_{1}, \quad I_{2} \bar{\varphi}_{1}=0 .
$$

For this to be in our sequence $\varphi_{k}$, we must have $s-4(k-1)=-s$ for some $k$. For some integer $n$, we therefore have

$$
s=2 n \quad \text { and } \quad \bar{\varphi}_{1}=\varphi_{n+1}, \quad I_{2} \varphi_{n+1}=0 .
$$

In this case, $\varphi_{k}, k=1, \ldots, n+1$, define a finite dimensional representation of the algebra. For other values of $s$, the representation is infinite.

The representations obtained in this way are deformations of representations of $\operatorname{sl}(2, \mathbb{C}$ ) (given in the Appendix), which correspond to the reduction $c_{1}=0$ (which is $m=-1$ ). In this reduction, the eigenvalue reduces to the value $\lambda_{-1,2 n}=\frac{1}{4} n(n+1)$. When $c_{1}=0, I_{2}$ reduces to $\mathbf{F}^{2}$, so our sequence $\varphi_{1}, \varphi_{2}, \ldots$ reduces to $\psi_{1}^{n}, \psi_{3}^{n}, \ldots$, jumping in steps of 2 . We thus retrieve only $n+1$ of the $2 n+1$ vectors of the highest weight representation.

Example 1 (The case $n=2$ ). Here we have

$$
\varphi_{1}=x y^{p}, \quad \varphi_{2}=4(3 y-1+4 p(y-1)) y^{p}, \quad \varphi_{3}=8(y-1)^{2}\left(3+16 p+16 p^{2}\right) y^{p},
$$

where $m=-1+4 p$. When $p=0$, these reduce to $\psi_{1}^{2}, \psi_{3}^{2}$ and $\psi_{5}^{2}$, given in the Appendix.

\section{Conclusions}

The main message of this paper is that super-integrability in a quantum system leads directly and explicitly to the construction of eigenfunctions. This leads to exact solvability, which is much stronger than just complete integrability.

The examples presented here are fairly simple, but the general approach can be used for any super-integrable system. The examples presented here are associated with the Laplace-Beltrami operator of a 2-dimensional space of constant curvature. The main feature used was the existence of a large number of symmetries of the underlying space. Using these it is possible to deform the Laplace-Beltrami operator, by adding a potential, in such a way that it possesses (generally) higher order (non-geometric) symmetries in the form of higher order commuting operators. This can be thought of as a 'ghost' of the previous geometric symmetry. These higher symmetries generate an algebra with nonlinear commutation relations, whose representations are built in a way analogous to the highest weight representations of Lie algebras. In 2 dimensions the symmetry algebra is very small, being just $s l(2, \mathbb{C})$ in our case (the 2-dimensional Euclidean algebra in the flat case), so the representations are very simple. In higher dimensions, the usual highest weight representations apply for the symmetry algebra of the Laplace-Beltrami operator. In this case there is no longer a single chain of eigenfunctions, so we can expect similar behaviour for the algebra of higher symmetries. 


\section{Appendix. Eigenfunctions of the Laplace-Beltrami operator}

We use the highest weight representations of the symmetry algebra $\operatorname{sl}(2, \mathbb{C})$ to construct eigenfunctions of $L_{b}$. Since $\mathbf{H}$ and $L_{b}$ commute, they share eigenfunctions and for $\mathbf{H}$ these are built by the highest weight construction. Furthermore, starting with any eigenfunction of $L_{b}$, we may use the symmetry algebra to construct further eigenfunctions (with the same eigenvalue). Since this eigenspace is invariant under the action of $\operatorname{sl}(2, \mathbb{C})$ (by construction), it can be decomposed into irreducible components, which are just weight spaces. Therefore all eigenfunctions of $L_{b}$ can be written as linear combinations of those we construct below.

A highest weight vector $\psi_{1}^{n}$, of weight $2 n$, satisfies

$$
\mathbf{E} \psi_{1}^{n}=0, \quad \mathbf{H} \psi_{1}^{n}=2 n \psi_{1}^{n},
$$

which constitute a pair of partial differential equations for the eigenfunction. These are compatible on the zeros of the differential operator $\mathbf{E}$, since

$$
\mathbf{H} \mathbf{E} \psi_{1}^{n}-\mathbf{E} \mathbf{H} \psi_{1}^{n}=2 \mathbf{E} \psi_{1}^{n}=0 .
$$

The specific form of $\psi_{1}^{n}$ depends upon $n$ and upon the choice of representation for $\operatorname{sl}(2, \mathbb{C})$. However, the general structure of the representation is independent of this specific form, being a consequence only of the commutation relations (4) (see [7]).

Defining $\psi_{r}^{n}=\mathbf{F}^{r-1} \psi_{1}^{n}$, the commutation relations imply:

$$
\mathbf{H} \psi_{r}^{n}=2(n+1-r) \psi_{r}^{n}, \quad \mathbf{E} \psi_{r}^{n}=(r-1)(2 n+2-r) \psi_{r-1}^{n} .
$$

Our definition of Laplace-Beltrami operator $L_{b}$ as Casimir operator (5) implies that

$$
L_{b} \psi_{r}^{n}=\frac{1}{4} n(n+1) \psi_{r}^{n}, \quad \text { for all } r, n \text {. }
$$

We can also construct a 3-point recursion relation between these eigenfunctions, but this is explicitly dependent upon the form of the operators. Let

$$
\mathbf{H}=h_{1} \partial_{z_{1}}+h_{2} \partial_{z_{2}}, \quad \mathbf{E}=e_{1} \partial_{z_{1}}+e_{2} \partial_{z_{2}}, \quad \mathbf{F}=f_{1} \partial_{z_{1}}+f_{2} \partial_{z_{2}},
$$

where $h_{i}$, etc are functions of the coordinates $z_{i}$. We have (with $\mu_{r}$ and $a_{r}$ defined by (14))

$$
\left.\begin{array}{l}
\mathbf{H} \psi_{r}^{n}=\mu_{r} \psi_{r}^{n}, \\
\mathbf{E} \psi_{r}^{n}=a_{r} \psi_{r-1}^{n}
\end{array}\right\} \Rightarrow\left(\begin{array}{l}
\partial_{z_{1}} \psi_{r}^{n} \\
\partial_{z_{2}} \psi_{r}^{n}
\end{array}\right)=\frac{1}{h_{1} e_{2}-e_{1} h_{2}}\left(\begin{array}{cc}
e_{2} & -h_{2} \\
-e_{1} & h_{1}
\end{array}\right)\left(\begin{array}{c}
\mu_{r} \psi_{r}^{n} \\
a_{r} \psi_{r-1}^{n}
\end{array}\right) .
$$

The relation $\psi_{r+1}^{n}=\mathbf{F} \psi_{r}^{n}$ then implies that

$$
\psi_{r+1}^{n}=\frac{f_{1} e_{2}-e_{1} f_{2}}{h_{1} e_{2}-e_{1} h_{2}} \mu_{r} \psi_{r}^{n}+\frac{h_{1} f_{2}-f_{1} h_{2}}{h_{1} e_{2}-e_{1} h_{2}} a_{r} \psi_{r-1}^{n} .
$$

When this is singular the representation reduces to 1 dimension.

In the case of our vector field representation (3), this takes explicit form

$$
\psi_{r+1}^{n}=2(n-r+1) \sqrt{\frac{y}{x}} \psi_{r}^{n}+(r-1)(2 n+2-r)\left(\frac{y-1}{x}\right) \psi_{r-1}^{n},
$$

with

$$
\psi_{1}^{n}=\sqrt{x^{n}}, \quad \psi_{2}^{n}=2 n \sqrt{x^{n-1} y} .
$$

When $n$ is an integer, these representations are of finite dimension $2 n+1$ and irreducible, but infinite dimensional otherwise. For instance, when $n=2$, we have

$$
\begin{aligned}
& \psi_{1}^{2}=x, \quad \psi_{2}^{2}=4 \sqrt{x y}, \quad \psi_{3}^{2}=4(3 y-1), \\
& \psi_{4}^{2}=24(y-1) \sqrt{\frac{y}{x}}, \quad \psi_{5}^{2}=\frac{24(y-1)^{2}}{x} .
\end{aligned}
$$




\section{References}

[1] Boyer C.P., Kalnins E.G., Winternitz P., Completely integrable relativistic Hamiltonian systems and separation of variables in Hermitian hyperbolic spaces, J. Math. Phys. 24 (1983), 2022-2034.

[2] Daskaloyannis C., Quadratic Poisson algebras of two-dimensional classical superintegrable systems and quadratic associative algebras of quantum superintegrable systems, J. Math. Phys. 42 (2001), 1100-1119, math-ph/0003017.

[3] Fordy A.P., Symmetries, ladder operators and quantum integrable systems, Glasg. Math. J. 47 (2005), $65-75$.

[4] Fordy A.P., Darboux related quantum integrable systems on a constant curvature surface, J. Geom. Phys. 56 (2006), 1709-1727.

[5] Gilmore R., Lie groups, Lie algebras and some of their applications, Wiley, New York, 1974.

[6] Harnad J., Vinet L., Yermolayeva O., Zhedanov A., Two-dimensional Krall-Sheffer polynomials and integrable systems, J. Phys. A: Math. Gen. 34 (2001), 10619-10625.

[7] Humphreys J.E., Introduction to Lie algebras and representation theory, Springer-Verlag, Berlin, 1972.

[8] Infeld L., Hull T., The factorization method, Rev. Modern Phys. 23 (1951), 21-68.

[9] Kalnins E.G., Kress J.M., Pogosyan G.S., Miller W.Jr., Completeness of superintegrability in twodimensional constant-curvature spaces, J. Phys. A: Math. Gen. 34 (2001), 4705-4720, math-ph/0102006.

[10] Kalnins E.G., Miller W.Jr., Hakobyan Ye.M., Pogosyan G.S., Superintegrability on the two-dimensional hyperboloid. II, J. Math. Phys. 40 (1999), 2291-2306, quant-ph/9907037.

[11] Kalnins E.G., Miller W.Jr., Pogosyan G.S., Exact and quasiexact solvability of second-order superintegrable quantum systems. I. Euclidean space preliminaries, J. Math. Phys. 47 (2006), 033502, 30 pages, math-ph/0412035.

[12] Krall H.L., Sheffer I.M., Orthogonal polynomials in two variables, Ann. Mat. Pura Appl. (4) 76 (1967), $325-376$.

[13] Kuznetsov V.B., Hidden symmetry of the quantum Calogero-Moser system, Phys. Lett. A 218 (1996), 212-222, solv-int/9509001.

[14] Tempesta P., Turbiner A.V., Winternitz P., Exact solvability of superintegrable systems, J. Math. Phys. 42 (2001), 4248-4257, hep-th/0011209.

[15] Vinet L., Zhedanov A., Two-dimensional Krall-Sheffer polynomials and quantum systems on spaces of constant curvature, Lett. Math. Phys. 65 (2003), 83-94. 